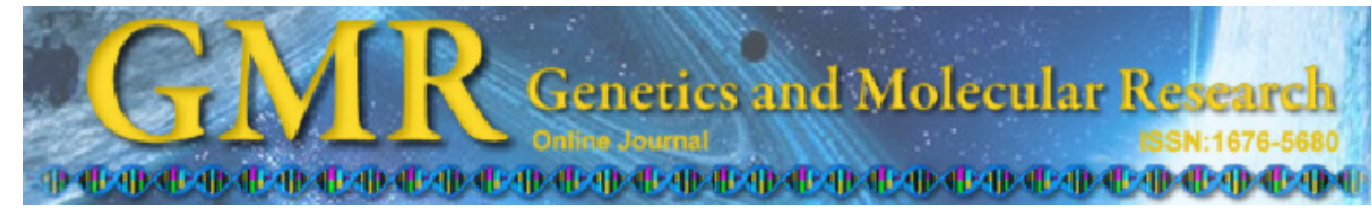

\title{
Genetic analysis of differentiation of T-helper lymphocytes
}

\author{
Q. Wang ${ }^{1}$, M. Li ${ }^{2}$, L.C. Xia ${ }^{3}$, G. Wen ${ }^{4}$, H. $\mathrm{Zu}^{5}$ and M. Gao ${ }^{6}$ \\ ${ }^{1}$ Department of Mathematics, University of Southern California, \\ Los Angeles, CA, USA \\ ${ }^{2}$ Department of Biomedical Engineering, Peking University, \\ Beijing, P.R. China \\ ${ }^{3}$ Department of Biological Sciences, University of Southern California, \\ Los Angeles, CA, USA \\ ${ }^{4}$ Department of Epidemiology, University of Southern California, \\ Los Angeles, CA, USA \\ ${ }^{5}$ Department of Electrical Engineering, University of Southern California, \\ Los Angeles, CA, USA \\ ${ }^{6}$ Department of Chemical Engineering and Materials, \\ University of Southern California, Los Angeles, CA, USA \\ Corresponding author: Q. Wang \\ E-mail: qixin.wang@usc.edu
}

Genet. Mol. Res. 12 (2): 972-987 (2013)

Received December 5, 2012

Accepted February 19, 2013

Published April 2, 2013

DOI http://dx.doi.org/10.4238/2013.April.2.13

ABSTRACT. In the human immune system, T-helper cells are able to differentiate into two lymphocyte subsets: Th1 and Th2. The intracellular signaling pathways of differentiation form a dynamic regulation network by secreting distinctive types of cytokines, while differentiation is regulated by two major gene loci: T-bet and GATA-3. We developed a system dynamics model to simulate the differentiation and re-differentiation process of T-helper cells, based on gene expression levels of T-bet and GATA-3 during differentiation of these cells. We arrived at three ultimate states of the model and came to the conclusion that cell differentiation potential exists as long as the system dynamics is at an unstable equilibrium point; the T-helper cells will no 
longer have the potential of differentiation when the model reaches a stable equilibrium point. In addition, the time lag caused by expression of transcription factors can lead to oscillations in the secretion of cytokines during differentiation.

Key words: Genetic analysis; System dynamics model; Th1-Th2; Equilibrium point

\section{INTRODUCTION}

CD4 T-helper cells play a crucial role in immune reactions and are ubiquitously known as a significant component of the human immune system. Human T-helper cells are further categorized into two subpopulations: Th1 and Th2 cells (Romagnani, 1992). The signaling transduction network that regulates Th1-Th2 differentiation is a typical dynamic network system.

In their process of differentiation, Th1 cells secrete cytokines such as TNF-beta, IL12 and IL-18, as well as IFN-gamma (Gately et al.,1998; Viola and Rao, 1999; Glimcher and Murphy, 2000; Nakanishi et al., 2001), while Th2 cells produce IL-4 (massively), IL-5, IL-6, IL-9, and IL-10 (Romagnani, 2000; O'Garra et al., 2000). Through the secretion of various cytokines, they function correctly as helpers in immune responses of distinct types.

With ongoing breakthroughs in research on T cells, the mechanism of Th1 and Th2 differentiation has unfolded gradually. CD4+ cells, when remaining undifferentiated, only secrete a tiny amount of cytokines and they are labeled Th0 cells or naive T-helper precursor cells (Lohning et al., 2002; Murphy and Reiner, 2002; Agnello et al., 2003; Wang et al., 2007a). Of the two differentiation paths, they will finally choose one, and their choice is decided on the external environment (various cytokines), mainly by IFN-gamma and IL-4 (O'Garra and Arai, 2000; Mullen et al., 2002; Szabo et al., 2003).

External IFN-gamma can induce Th0 to differentiate into Th1. First, IFN-gamma binds to its receptors on the outer surface of the cell membrane, which activates the signal molecule STAT-1 and triggers its nuclear translocation (Szabo et al., 2000; Lighvani et al., 2001). Afterwards, STAT-1 activates the transcription factor T-bet, which plays a dominant role in regulating the differentiation of T-helper cells (Chakir et al., 2003). When T-bet binds to the gene that can be transcribed and translated into IFN-gamma, histone acetylation and DNA methylation are catalyzed and the gene is modified, hence the alteration of its accessibility, which leads to chromatin remodeling (Robinson and Lloyd, 2002; Lovett-Racke et al., 2004; Mariani et al., 2004; Wang et al., 2007b). Generally, the IFN-gamma gene undergoes morphological changes so that it can be transcribed more easily. As the production of IFN-gamma is augmented, part of it is transported out of the cell and once again activates the IFN-gamma/Tbet pathway to establish a positive feedback loop (Ouyang et al., 2000; Ho and Glimcher, 2002; Wang et al., 2008). Moreover, T-bet can also influence GATA-3's function in inhibiting the transcription of IL-4 (Szabo et al., 2000).

Besides, IL-12, mainly secreted by dendritic cells, monocytes and macrophages, is another crucial cytokine that induces Th1 differentiation. In the early differentiation stage, IL-12 activates STAT-4 via the receptor complex on the cell membrane (Gately et al., 1998). STAT4 can simultaneously affect the IFN-gamma and T-bet genes (Robinson and Lloyd, 2002; Lovett-Racke et al., 2004), leading to more combination of IFN-gamma and T-bet, which 
boosts the translation of IFN-gamma and its production. Following a certain period of time after the onset of differentiation, differentiation can proceed smoothly even without IL-12, because the necessity of its promoting effect decreases with time (Lohning et al., 2002; Mariani et al., 2004). Note that IL-12 is not an essential cytokine: when the IL-12 gene of T-helper cells is knocked out, these cells can still secrete IFN-gamma, but at much lower levels than normal (Jankovic et al., 2002).

External IL-4 is able to induce Th0 to differentiate into Th2 (Ranganath and Murphy, 2001; Lametschwandtner et al., 2004). Initially, IL-4 binds to its receptors on the outer surface of cell membrane and activates STAT-6 and finally GATA-3 (Ranganath and Murphy, 2001; Zhou et al., 2001; Smits et al., 2001; Lametschwandtner et al., 2004). Activated GATA-3 then changes the accessibility of IL-4 and thereby stimulates the transcription of IL-4, forming a positive feedback loop. GATA-3 can also inhibit the function of T-bet in the same way as T-bet does (Ranganath and Murphy, 2001; Zhou et al., 2001; Smits et al., 2001).

The process of differentiation can be divided into two stages: polarization and differentiation. Polarization is defined as the structural transformation of Th0 towards either subpopulation of Th1 and Th2 prior to the completion of differentiation. The structural change of Th0 at this stage is reversible, which means the cell is still able to switch from Th1 to Th2 or backwards, if its culture environment is properly altered. However, a Th2 cell with thorough differentiation cannot possibly transform into a Th1 cell, even when a large quantity of IFN-gamma is added to the culture environment or a high level of T-bet is expressed in cells. Nevertheless, the latter method does generate a special type of cell that produces both IL-4 and IFN-gamma, which is presumably due to the subtle change where the IL-4 gene with chromatin remodeling is no longer inhibited by T-bet (Smits et al., 2001; Zhou and Ouyang, 2003; Yates et al., 2004). This process is shown in Figure 1.

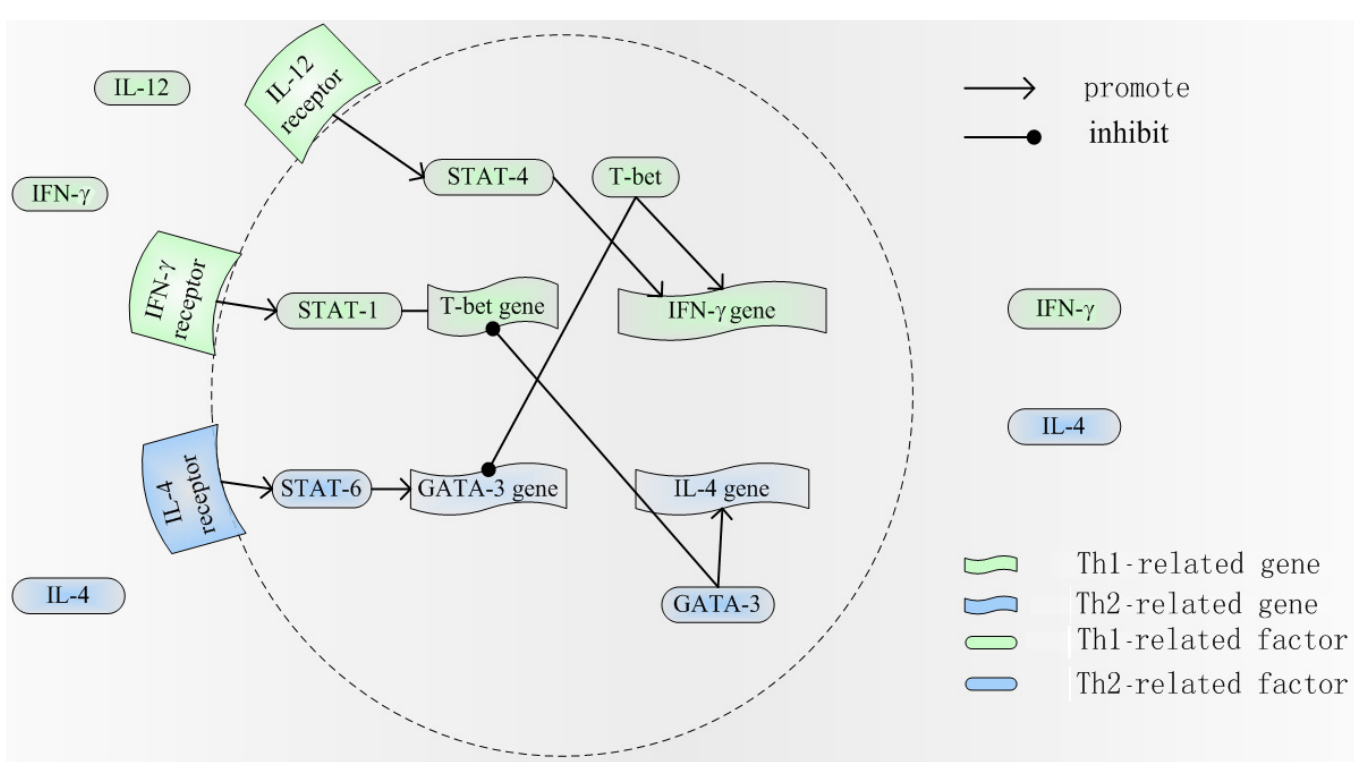

Figure 1. Differentiation-related intracellular signaling pathway for Th1 and Th2 cells. 


\section{MATERIAL AND METHODS}

\section{System dynamics model of differentiation process}

\section{Differentiation of Th1}

System dynamics models have a wide range of applications in the field of biological regulatory networks. We developed a lag-system dynamics model to simulate the differentiation process of Th0 cell to Th1/Th2 cells, symbols and definitions of this model are shown in Table 1.

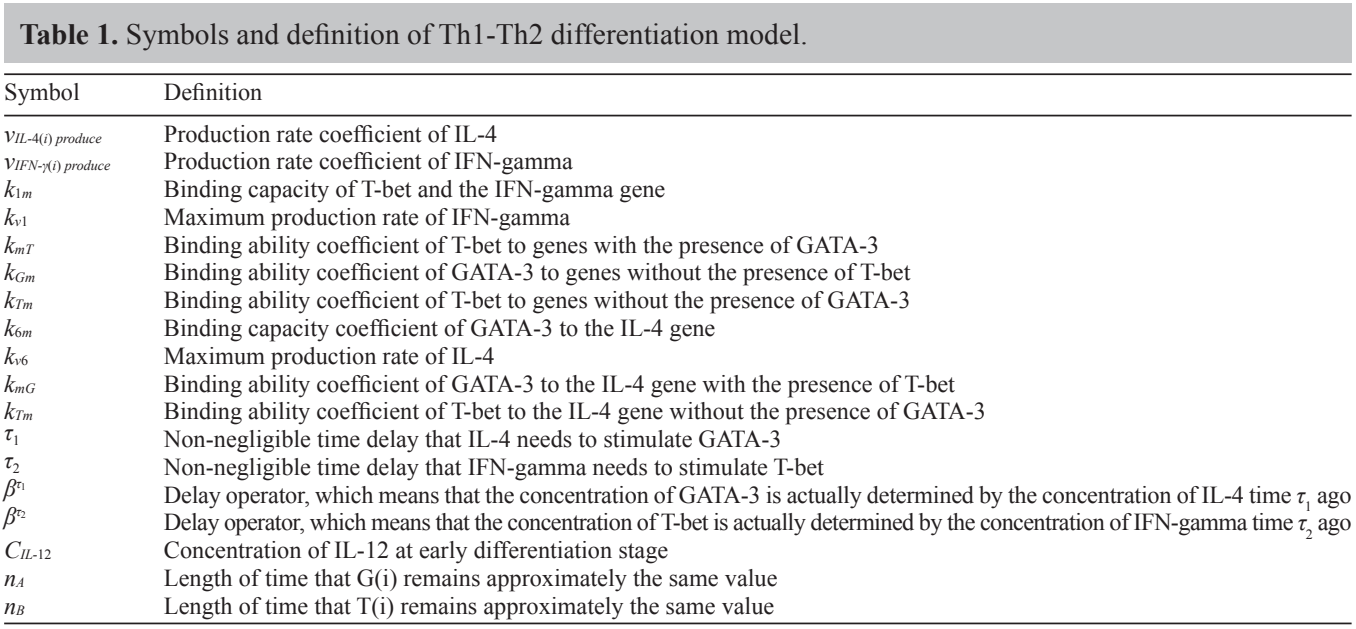

First, we assume that all genes are transcribed continuously. If we define the concentration of T-bet as T(i) at time $\mathrm{i}$, the production rate of IFN-gamma can be modeled as:

$$
v_{I F N-\gamma(i) \text { produce }}=\frac{k_{v 1} T(i)}{k_{1 m}+T(i)}
$$

Equation 1 apparently shows that the production rate of IFN-gamma increases nonlinearly as the concentration of T-bet rises, and production rate of IFN-gamma is almost directly proportional to $\mathrm{T}(\mathrm{i})$, when the concentration of T-bet is extremely low.

Under the conditions that the concentration of T-bet is elevated to an exceptionally high level, the production rate of IFN-gamma approaches a fixed value. The production rate of T-bet is expressed by:

$$
v_{T(i) \text { produce }}=\frac{k_{I F} \beta^{\tau_{2}} v_{I F N-\gamma(i) \text { produce }}}{1+\frac{k_{I F}}{k_{I F T}} \beta^{\tau_{2}} v_{I F N-\gamma(i) \text { produce }}}
$$


If we substitute Equation 1 into Equation 2, we will arrive at:

$$
v_{T(i) \text { produce }}=\frac{k_{I F} \frac{k_{v 1} \beta^{\tau_{2}} T(i)}{k_{1 m}+\beta^{\tau_{2}} T(i)}}{1+\frac{k_{I F}}{k_{I F T}} * \frac{k_{v 1} \beta^{\tau_{2}} T(i)}{k_{1 m}+\beta^{\tau_{2}} T(i)}} \quad \text { (Equation 3) }
$$

which can be simplified as follows:

$$
v_{T(i) \text { produce }}=\frac{k_{I F} k_{v 1} k_{I F T} \beta^{\tau_{2}} T(i)}{k_{1 m} k_{I F T}+\left(k_{I F T}+k_{I F} k_{v 1}\right) \beta^{\tau_{2}} T(i)} \quad \text { (Equation 4) }
$$

Since GATA-3 can affect the binding of T-bet to the IFN-gamma gene, the binding rate of T-bet and IFN-gamma is given by the following formulas:

$$
\begin{gathered}
v_{T(i) b i n d}=\frac{k_{v T} T(i)}{k_{m T}+T(i)} \\
k_{m T}=k_{T m}\left(1+\frac{G(i) I_{B}}{k_{G m}}\right)\left(1+\frac{k_{c} C_{I L-12}}{i}\right), I_{B}=\left\{\begin{array}{ll}
1 & , i \notin B \\
0 & , i \in B
\end{array} \quad B=\left\{i \mid T(i)=\frac{1}{n_{B}} \sum_{t=i-n_{B}}^{i-1} \beta^{r} T(i)\right\} \quad\right. \text { (Equation 6) }
\end{gathered}
$$

As G(i) increases, it inhibits T-bet more substantially, and when less GATA-3 is produced, more T-bet will bind to the IFN-gamma gene. If T(i) remains at approximately the same value for a rather long period of time, it means that the Th cells have reached the stage of complete differentiation. Under such circumstances, the process of differentiation is irreversible, even when a large quantity of IL-4 is added to the culture environment or a high level of GATA-3 is expressed in cells; in other words, the change in G(i) cannot affect the binding of T-bet to the IFN-gamma gene. In the early stage of Th1 differentiation, IL-12 is able to simultaneously stimulate both the IFN-gamma and T-bet gene through IL-12 to STAT-4 transduction pathway and accelerates IFN-gamma gene transcription. As the process of differentiation proceeds, the promoting effect of IL-12 diminishes, until differentiation continues independently, even in the absence of IL-12.

Next, we substitute Equation 6 into Equation 5 to get:

$$
v_{T(i) \text { bind }}=\frac{k_{v T} T(i)}{k_{T m}\left(1+\frac{G(i) I_{B}}{k_{G m}}\right)\left(1+\frac{k_{c} C_{I L-12}}{i}\right)+T(i)} \quad \text { (Equation 7) }
$$

We then assume that T-bet will not degrade before binding to the IFN-gamma gene, based on what we have: 


$$
\frac{d T(i)}{d t}=v_{T(i) \text { produce }}-v_{T(i) \text { bind }}
$$

We substitute Equations 4 and 7 into Equation 8, so we then have:

$$
\frac{d T(i)}{d t}=\frac{k_{I F} k_{v 1} k_{I F T} \beta^{\tau_{2}} T(i)}{k_{1 m} k_{I F T}+\left(k_{I F T}+k_{I F} k_{v 1}\right) \beta^{\tau_{2}} T(i)}-\frac{k_{v T} T(i)}{k_{I m}\left(1+\frac{G(i) I_{B}}{k_{G m}}\right)\left(1+\frac{k_{c} C_{I L-12}}{i}\right)+T(i)} \quad \text { (Equation 9) }
$$

\section{Differentiation of Th2}

We define G(i) as the concentration of GATA-3 in cells at moment $i$, and the production rate of IL-4 is modeled as:

$$
v_{I L-4(i) \text { produce }}=\frac{k_{v 6} G(i)}{k_{6 m}+G(i)}
$$

Equation 10 shows that the production rate of IL-4 increases non-linearly with an increase in GATA-3 concentration. When G(i) is close to 0 , the production rate of IL-4 is almost directly proportional to G(i), provided that the concentration of GATA-3 is extremely low.

Under the conditions where the concentration of GATA-3 is elevated to an exceptionally high level, the production rate of IL-4 approaches a fixed value. Due to the fact that the transduction pathway is comparatively long, the production rate of GATA-3 is expressed by:

$$
v_{G(i) \text { produce }}=\frac{k_{I L} \beta^{r_{1}} v_{I L-4(i) \text { produce }}}{1+\frac{k_{I L}}{k_{I L G}} \beta^{r_{1}} v_{I L-4(i) \text { produce }}}
$$

When we substitute Equation 10 into Equation 11, we then arrive at:

$$
v_{G(i) \text { produce }}=\frac{k_{I L} \frac{k_{v 6} \beta^{\tau_{1}} G(i)}{k_{6 m}+\beta^{\tau_{1}} G(i)}}{1+\frac{k_{I L}}{k_{I L G}} * \frac{k_{v 6} \beta^{\tau_{1}} G(i)}{k_{6 m}+\beta^{r_{1}} G(i)}}
$$

and its simplified form is:

$$
v_{G(i) \text { produce }}=\frac{k_{I L} k_{v 6} k_{I L G} \beta^{\tau_{1}} G(i)}{k_{6 m} k_{I L G}+\left(k_{I L G}+k_{I L} k_{v 6}\right) \beta^{\tau_{1}} G(i)} \text { (Equation 13) }
$$


Since T-bet can affect the binding of GATA-4 to the IL-4 gene, the binding rate of GATA-4 and IL-4 is given by the following formulas:

$$
\begin{gathered}
v_{G(i) \text { bind }}=\frac{k_{1 G} G(i)}{k_{m G}+G(i)} \\
k_{m G}=k_{G m}\left(1+\frac{T(i) I_{A}}{k_{T m}}\right), \quad I_{A}=\left\{\begin{array}{ll}
1 & , i \notin A \\
0 & , i \in A
\end{array} \quad A=\left\{i \mid G(i)=\frac{1}{n_{A}} \sum_{\tau=i-n_{A}}^{i-1} \beta^{\tau} G(i)\right\}\right.
\end{gathered}
$$

As T(i) increases, it inhibits GATA-3 more substantially, and when less T-bet is produced, more GATA-3 will bind to the IL-4 gene. If G(i) remains approximately at the same value for a rather long period of time, it shows that the Th cells have reached the stage of complete differentiation. Under such circumstances, the process of differentiation is irreversible, even when a large quantity of IFN-gamma is added to the culture environment or a high level of T-bet is expressed in the cells; in other words, a change in T(i) can no longer affect the binding of GATA-3 to the IL-4 gene.

We then substitute Equation 15 into Equation 14 to get:

$$
v_{G(i) b i n d}=\frac{k_{v G} G(i)}{k_{G m}\left(1+\frac{T(i) I_{A}}{k_{T m}}\right)+G(i)}
$$

Next, we assume that GATA-3 will not degrade before binding to the IL-4 gene, based on what we have:

$$
\frac{d G(i)}{d t}=v_{G(i) \text { produce }}-v_{G(i) b i n d}
$$

We substitute Equations 13 and 16 into Equation 17, so we have:

$$
\frac{d G(i)}{d t}=\frac{k_{I L} k_{v 6} k_{I L G} \beta^{\tau_{1}} G(i)}{k_{6 m} k_{I L G}+\left(k_{I L G}+k_{I L} k_{v 6}\right) \beta^{\tau_{1}} G(i)}-\frac{k_{v G} G(i)}{k_{G m}\left(1+\frac{T(i) I_{A}}{k_{T m}}\right)+G(i)}
$$

Note that Th cells are almost unable to spontaneously secrete cytokines, except IFNgamma and IL-4, without the stimulation of other types of cytokines. Since this experiment is designed to culture Th cells in an environment devoid of cytokines, except IFN-gamma, IL-12 and IL-4, the possible existence and influence of other cytokines can be theoretically ignored. Thus, the differentiation of Th cells is modeled as: 


$$
\left\{\begin{array}{l}
\frac{d T(i)}{d t}=\frac{k_{I F} k_{v 1} k_{I F T} T\left(i-\tau_{2}\right)}{k_{1 m} k_{I F T}+\left(k_{I F T}+k_{I F} k_{v 1}\right) T\left(i-\tau_{2}\right)}-\frac{k_{v T} T(i)}{k_{T m}\left(1+G(i) I_{B} / k_{G m}\right)\left(1+k_{c} C_{I L-12} / i\right)+T(i)} \\
\frac{d G(i)}{d t}=\frac{k_{I L} k_{v 6} k_{I L G} G\left(i-\tau_{1}\right)}{k_{6 m} k_{I L G}+\left(k_{I L G}+k_{I L} k_{v 6}\right) G\left(i-\tau_{1}\right)}-\frac{k_{v G} G(i)}{k_{G m}\left(1+T(i) I_{A} / k_{T m}\right)+G(i)}
\end{array}\right.
$$

\section{Model analysis}

The completion of the differentiation process is reached when:

$$
\left\{\begin{array}{l}
\frac{k_{I F} k_{v 1} k_{I F T} T\left(i-\tau_{2}\right)}{k_{1 m} k_{I F T}+\left(k_{I F T}+k_{I F} k_{v 1}\right) T\left(i-\tau_{2}\right)}-\frac{k_{v T} T(i)}{k_{T m}\left(1+G(i) I_{B} / k_{G m}\right)+T(i)}=0 \\
\frac{k_{I L} k_{v 6} k_{I L G} G\left(i-\tau_{1}\right)}{k_{6 m} k_{I L G}+\left(k_{I L G}+k_{I L} k_{v 6}\right) G\left(i-\tau_{1}\right)}-\frac{k_{v G} G(i)}{k_{G m}\left(1+T(i) I_{A} / k_{T m}\right)+G(i)}=0
\end{array}\right.
$$

At this moment, limit points for the equations exist. When the cells are at the Th0 stage, we have $\mathrm{T}(\mathrm{i})=0$ and $\mathrm{G}(\mathrm{i})=0$. According to Equation 20, we then have:

$$
\left\{\begin{array}{l}
\frac{k_{I F} k_{v 1} k_{I F T} T\left(i-\tau_{2}\right)}{k_{1 m} k_{I F T}+\left(k_{I F T}+k_{I F} k_{v 1}\right) T\left(i-\tau_{2}\right)}-\frac{k_{v T} T(i)}{k_{T m}\left(1+G(i) I_{B} / k_{G m}\right)\left(1+k_{c} C_{I L-12} / i\right)+T(i)}=0 \\
\frac{k_{I L} k_{v 6} k_{I L G} G\left(i-\tau_{1}\right)}{k_{6 m} k_{I L G}+\left(k_{I L G}+k_{I I} k_{v 6}\right) G\left(i-\tau_{1}\right)}-\frac{k_{v G} G(i)}{k_{G m}\left(1+T(i) I_{A} / k_{I m}\right)+G(i)}=0
\end{array}\right.
$$

The differentiation of Th1 is considered to be completed when G(i) is close to 0. According to Equation 19, we then have:

$$
\begin{aligned}
& \lim _{G(i) \rightarrow 0} \frac{k_{I F} k_{v 1} k_{I F T} T\left(i-\tau_{2}\right)}{k_{1 m} k_{I F T}+\left(k_{I F T}+k_{I F} k_{v 1}\right) T\left(i-\tau_{2}\right)}-\frac{k_{v T} T(i)}{k_{T m}\left(1+G(i) I_{B} / k_{G m}\right)\left(1+k_{c} C_{I L-12} / i\right)+T(i)} \\
& =\frac{k_{I F} k_{v 1} k_{I F T} T(i)}{k_{1 m} k_{I F T}+\left(k_{I F T}+k_{I F} k_{v 1}\right) T(i)}-\frac{k_{v T} T(i)}{k_{T m}+T(i)} \\
& \Rightarrow \quad \frac{k_{I F} k_{v 1} k_{I F T} T(i)}{k_{1 m} k_{I F T}+\left(k_{I F T}+k_{I F} k_{v 1}\right) T(i)}-\frac{k_{v T} T(i)}{k_{T m}+T(i)}=0
\end{aligned}
$$

(Equation 22)

The solution is:

$$
T(i)=\frac{k_{I F} k_{v 1} k_{I F T} k_{I m}-k_{1 m} k_{I F T} k_{v T}}{k_{I F T} k_{v T}+k_{I F} k_{v 1} k_{v T}-k_{I F} k_{v 1} k_{I F T}}
$$

On the other hand, 


$$
\lim _{G(i) \rightarrow 0} \frac{k_{I L} k_{v 6} k_{I L G} G\left(i-\tau_{1}\right)}{k_{6 m} k_{I L G}+\left(k_{I L G}+k_{I L} k_{v 6}\right) G\left(i-\tau_{1}\right)}-\frac{k_{v G} G(i)}{k_{G m}\left(1+T(i) I_{A} / k_{T m}\right)+G(i)}=0
$$

Thus, one stable equilibrium point for Equation 19 is:

$$
P_{0}\left(G_{1}^{0}, T_{1}^{0}\right)=\left(0, \frac{k_{I F} k_{v 1} k_{I F T} k_{T m}-k_{1 m} k_{I F T} k_{v T}}{k_{I F T} k_{v T}+k_{I F} k_{v 1} k_{v T}-k_{I F} k_{v 1} k_{I F T}}\right)
$$

The process of Th2 differentiation is over when T(i) is close to 0. According to Equation 19, we then have:

$$
\begin{aligned}
& \lim _{T(i) \rightarrow 0} \frac{k_{I L} k_{v 6} k_{I L G} G\left(i-\tau_{1}\right)}{k_{6 m} k_{I L G}+\left(k_{I L G}+k_{I L} k_{v 6}\right) G\left(i-\tau_{1}\right)}-\frac{k_{v G} G(i)}{k_{G m}\left(1+T(i) I_{A} / k_{T m}\right)+G(i)} \\
= & \frac{k_{I L} k_{v 6} k_{I L G} G(i)}{k_{6 m} k_{I L G}+\left(k_{I L G}+k_{I L} k_{v 6}\right) G(i)}-\frac{k_{v G} G(i)}{k_{G m}+G(i)} \\
\Rightarrow \quad & \frac{k_{I L} k_{v 6} k_{I L G} G(i)}{k_{6 m} k_{I L G}+\left(k_{I L G}+k_{I L} k_{v 6}\right) G(i)}-\frac{k_{v G} G(i)}{k_{G m}+G(i)}=0
\end{aligned}
$$

The solution is:

$$
G(i)=\frac{k_{I L} k_{v 6} k_{I L G} k_{G m}-k_{6 m} k_{I L G} k_{v G}}{k_{I L G} k_{v G}+k_{I L} k_{v 6} k_{v G}-k_{I L} k_{v 6} k_{I L G}}
$$

On the other hand,

$$
\lim _{T(i) \rightarrow 0} \frac{k_{I F} k_{v 1} k_{I F T} T\left(i-\tau_{2}\right)}{k_{1 m} k_{I F T}+\left(k_{I F T}+k_{I F} k_{v 1}\right) T\left(i-\tau_{2}\right)}-\frac{k_{v T} T(i)}{k_{T m}\left(1+G(i) I_{B} / k_{G m}\right)+T(i)}=0
$$

Thus, another stable equilibrium point for Equation 19 is:

$$
P_{0}\left(G_{2}^{0}, T_{2}^{0}\right)=\left(\frac{k_{I L} k_{v 6} k_{I L G} k_{G m}-k_{6 m} k_{I L G} k_{v G}}{k_{I L G} k_{v G}+k_{I L} k_{v 6} k_{v G}-k_{I L} k_{v 6} k_{I L G}}, 0\right)
$$

Three equilibrium points in the system dynamics model are shown in Table 2. 
Table 2. Equilibrium points of system dynamic model.

\begin{tabular}{|c|c|c|}
\hline Differentiation state & Results & Stable status \\
\hline Th0 state & $P_{0}\left(G_{0}^{0}, T_{0}^{0}\right)=(0,0)$ & Unstable equilibrium point \\
\hline Th1 differentiation state & $P_{0}\left(G_{1}^{0}, T_{1}^{0}\right)=\left(0, \frac{k_{I F} k_{v i v} k_{I F} k_{T w}-k_{I m} k_{l F T} k_{v T}}{k_{I F T} k_{V T}+k_{I F}}\right)$ & Stable equilibrium point \\
\hline Th2 differentiation state & $P_{0}\left(G_{2}^{0}, T_{2}^{0}\right)=\left(\frac{k_{u L} k_{v G} k_{I L G} k_{v i m}-k_{6 m} k_{I L G} k_{v G}}{k_{I I G} k_{v G}+k_{H L} k_{v G} k_{v G}-k_{H L} k_{v G} k_{H L G}}, 0\right)$ & Stable equilibrium point \\
\hline
\end{tabular}

\section{RESULTS AND DISCUSSION}

\section{Simulation results of differentiation}

\section{Th1/Th2 differentiation process}

Mathematical modeling plays an important role in the analysis of a great deal of complicated experimental phenomena of biological systems (Xia and Zhou, 2007; Xia et al., 2011; Zhou and Nakhleh, 2011; Zu et al., 2012). Here, we discuss the relationship between differentiation potential and equilibrium points of the Th1-Th2 system dynamics model.

Figures 2 and 3 show the differentiation process of both Th1 and Th2 cells. When there is no stimulation of cytokines or other signals, Th0 cells may differentiate into Th1 or Th2 cells with equal probability. Cytokines (IFN-gamma, IL-4) can stimulate transcription factors (T-bet, GATA3 ), which results in a certain period of time delay that in turn brings about oscillation in the expression of T-bet and GATA-3 (the oscillation becomes more evident when the graph is enlarged), but they cannot influence the equilibrium point of the differential equation (i.e., the concentration when a steady state is reached). Therefore, the oscillation can explain the abnormity of the curves representing T-bet and GATA-3 (Chakir et al., 2003). Moreover, it will take the differentiation process a longer time to reach the stable point when more considerable oscillation occurs.

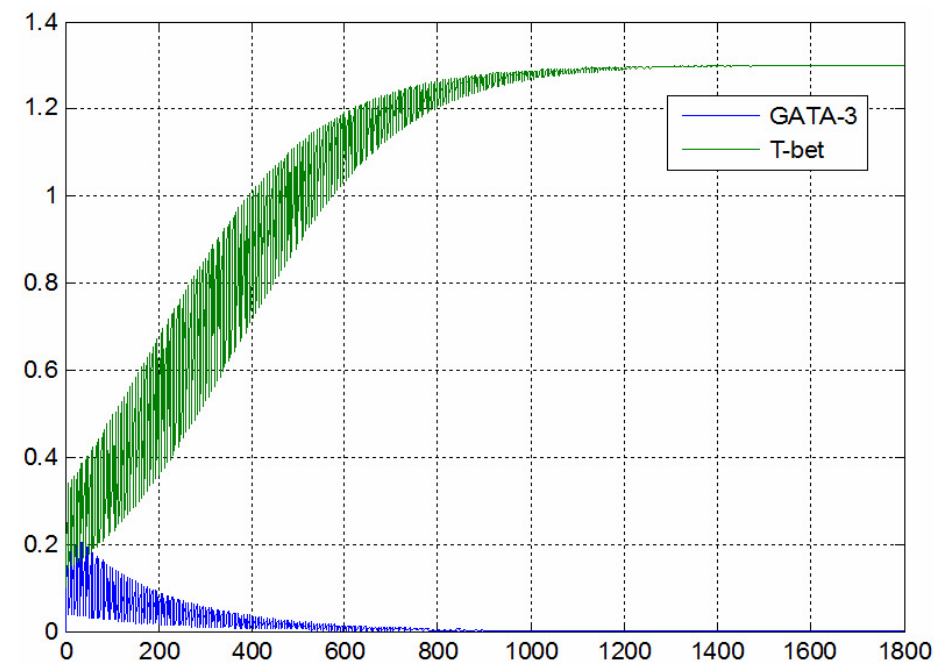

Figure 2. Expression level of major regulation gene in Th1 differentiation process. 


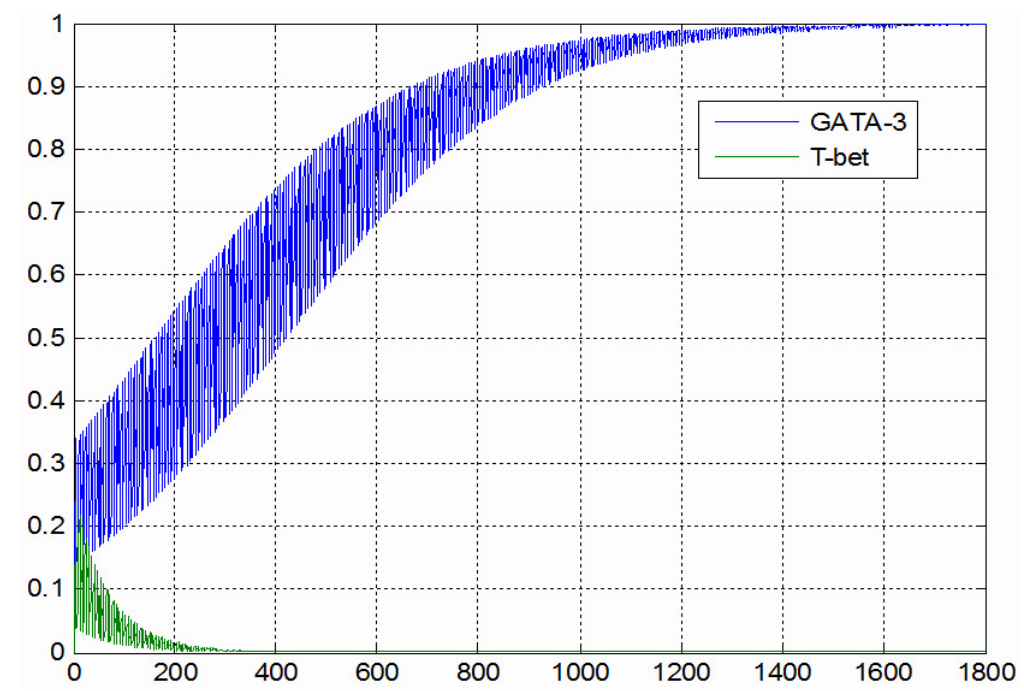

Figure 3. Expression level of major regulation gene in Th2 differentiation process.

\section{Th1/Th2 re-differentiation process}

In the middle of differentiation (Figure 4), when the expression level of T-bet has not yet reached a stable equilibrium point, increased expression of GATA-3 will lead to a decrease in T-bet expression, which means that re-differentiation occurs.

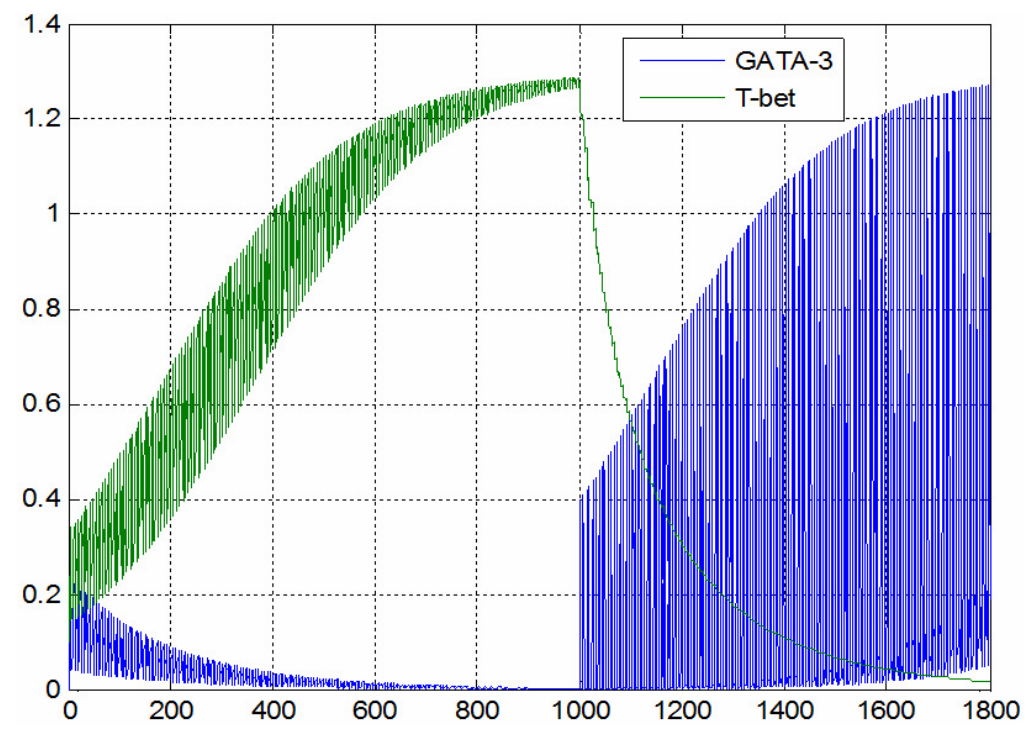

Figure 4. Expression level of major regulation gene in Th1 to Th2 re-differentiation process. 
In the middle of differentiation (Figure 5), when the expression level of GATA-3 has not yet reached its stable equilibrium point, an increase in T-bet expression will lead to a decrease in GATA-3 expression, which means that re-differentiation occurs.

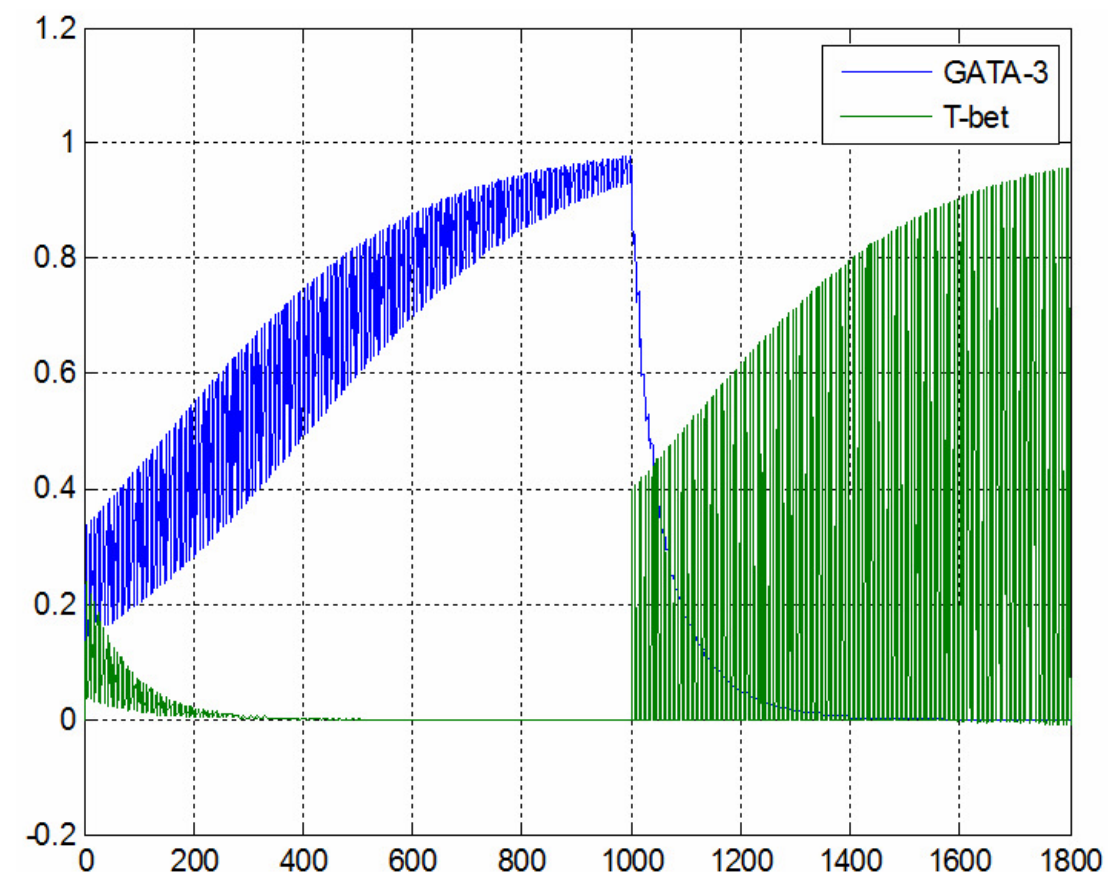

Figure 5. Expression level of major regulation gene in Th2 to Th1 re-differentiation process.

\section{Th1/Th2 cell without potential of differentiation}

After differentiation is complete (Figure 6), the expression level of T-bet cannot be affected even when a high level of GATA-3 is expressed in cells. However, by transiently increasing the expression level of GATA-3, it is possible to obtain re-differentiated cells that can transiently secrete IFN-gamma and IL-4. The mechanism is: after G(i) and T(i) remain stable for a while, Equation 19 transforms into Equation 26 [G(i) and T(i) cannot affect each other]; then we have:

$$
\left\{\begin{array}{l}
\frac{d T(i)}{d t}=\frac{k_{I F} k_{v 1} k_{I F T} T\left(i-\tau_{2}\right)}{k_{1 m} k_{I F T}+\left(k_{I F T}+k_{I F} k_{v 1}\right) T\left(i-\tau_{2}\right)}-\frac{k_{v T} T(i)}{k_{T m}+T(i)} \\
\frac{d G(i)}{d t}=\frac{k_{I L} k_{v 6} k_{I L G} G\left(i-\tau_{1}\right)}{k_{6 m} k_{I L G}+\left(k_{I L G}+k_{I L} k_{v 6}\right) G\left(i-\tau_{1}\right)}-\frac{k_{v G} G(i)}{k_{G m}+G(i)}
\end{array}\right.
$$




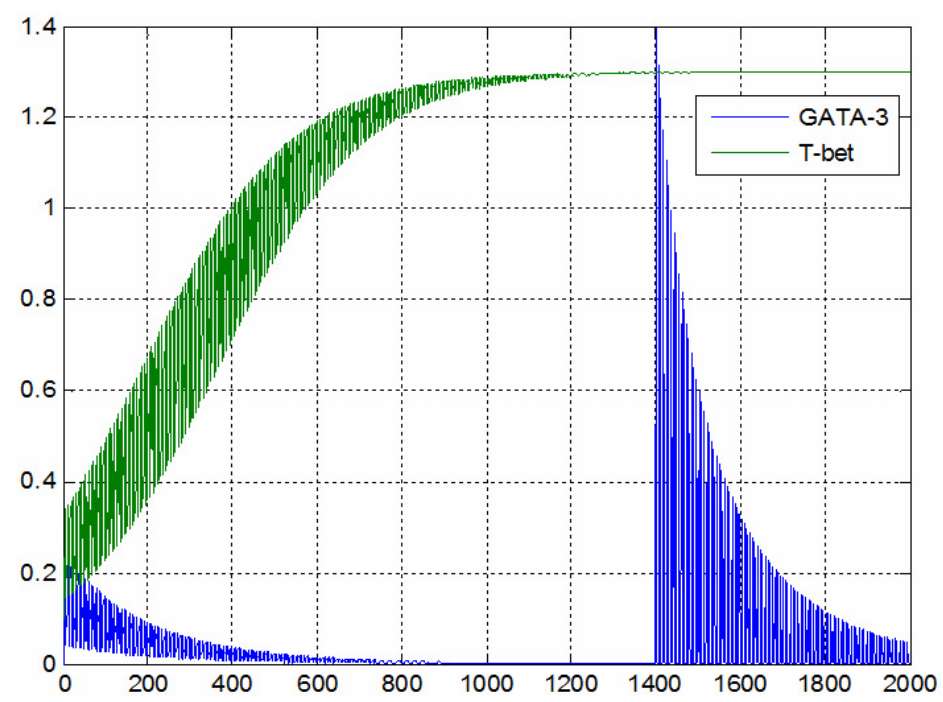

Figure 6. Th1 cell without potential of differentiation.

Due to the reconstruction of the chromosome, the binding of T-bet to the IFN-gamma gene is not influenced by GATA-3, and therefore, the production rates of IFN-gamma and Tbet remain constant. Under these circumstances, the fluctuation of G(i) cannot affect T(i), even when there is a high level of GATA-3 in cells.

Similarly, after differentiation is complete (Figure 7), the expression level of GATA-3 cannot be affected even when a high level of T-bet is expressed in cells, but it is possible to obtain re-differentiated cells that can transiently secrete IFN-gamma and IL-4.

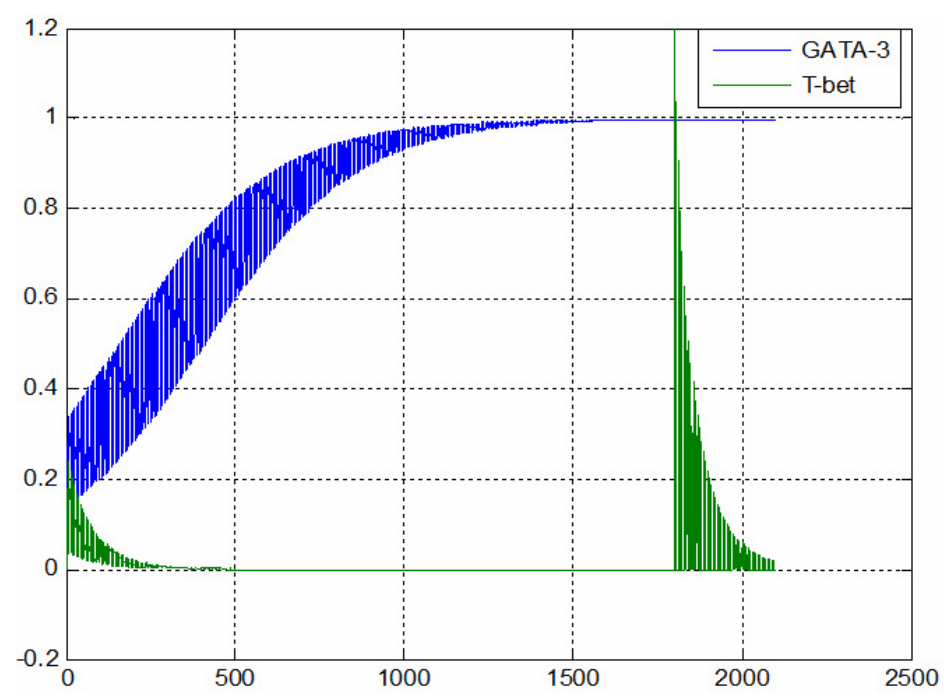

Figure 7. Th2 cell without potential of differentiation. 


\section{Phase trajectory}

We can see from Figures 8 and 9 that when the lag increases, commotion is enhanced. The Figures show that oscillation does not exist when either one of GATA-3 and T-bet is obviously superior to the other in quantity (comparatively stable state of differentiation), whereas in an unstable state, the phase trajectory oscillates more considerably as the delay order increases. In addition, the curve oscillates dramatically when the level of expression of T-bet is low.

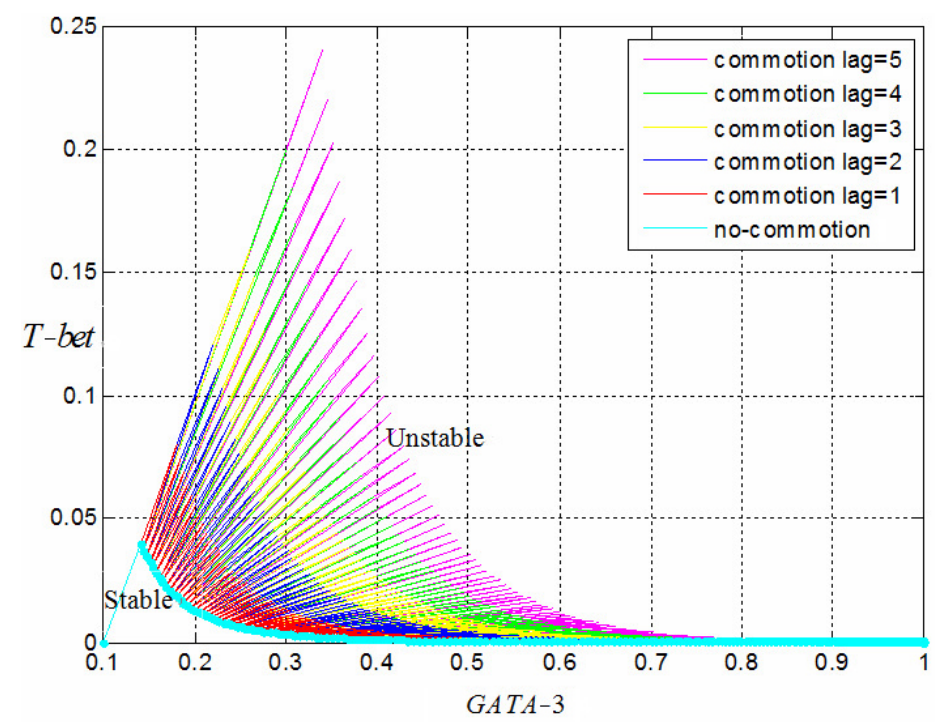

Figure 8. Phase trajectory curve of Th2 differentiation.

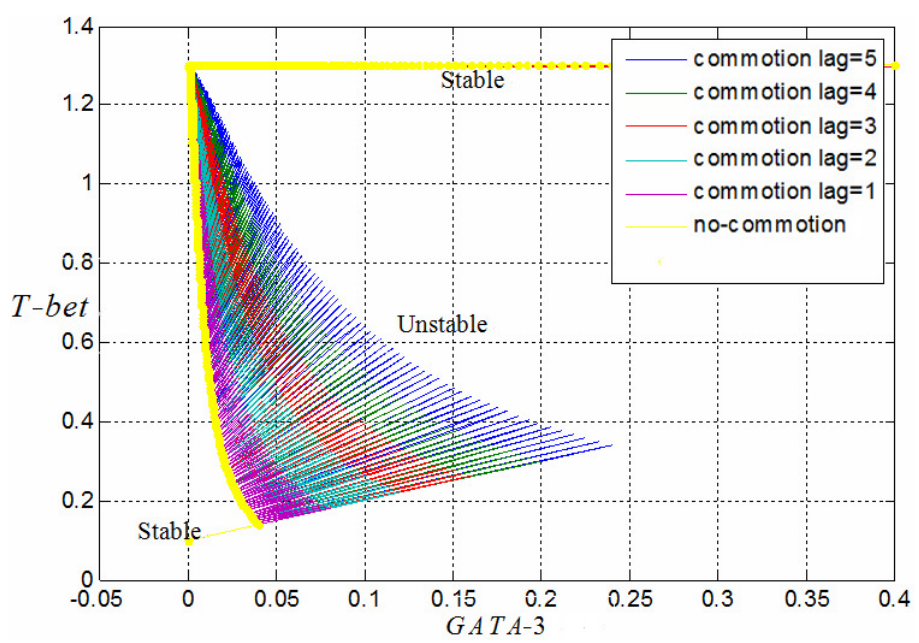

Figure 9. Phase trajectory curve of Th1 differentiation. The yellow curve in this figure is the phase trajectory of differentiation ignoring the time lag in the transduction pathway. 


\section{CONCLUSION}

T-helper lymphocytes have the potential to differentiate when a system dynamics model shows the expression of major control genes at an unstable equilibrium point. The T-helper lymphocyte will no longer have differentiation potential when the model evolution reaches a stable equilibrium point. In addition, the time lag, caused by the expression of transcription factors, can lead to an oscillation in the secretion of cytokines during the differentiation process.

\section{REFERENCES}

Agnello D, Lankford CS, Bream J, Morinobu A, et al. (2003). Cytokines and transcription factors that regulate T helper cell differentiation: new players and new insights. J. Clin. Immunol. 23: 147-161.

Chakir H, Wang H, Lefebvre DE, Webb J, et al. (2003). T-bet/GATA-3 ratio as a measure of the Th1/Th2 cytokine profile in mixed cell populations: predominant role of GATA-3. J. Immunol. Methods 278: 157-169.

Gately MK, Renzetti LM, Magram J, Stern AS, et al. (1998). The interleukin-12/interleukin-12-receptor system: role in normal and pathologic immune responses. Annu. Rev. Immunol. 16: 495-521.

Glimcher LH and Murphy KM (2000). Lineage commitment in the immune system: the T helper lymphocyte grows up. Genes Dev. 14: 1693-1711.

Ho C and Glimcher LH (2002). Transcription: tantalizing times for T cells. Cell 109: S109-S120.

Jankovic D, Kullberg MC, Hieny S, Caspar P, et al. (2002). In the absence of IL-12, CD4(+) T cell responses to intracellular pathogens fail to default to a Th2 pattern and are host protective in an IL-10(-/-) setting. Immunity 16: 429-439.

Lametschwandtner G, Biedermann T, Schwarzler C, Gunther C, et al. (2004). Sustained T-bet expression confers polarized human TH2 cells with TH1-like cytokine production and migratory capacities. J. Allergy Clin. Immunol. 113: 987-994.

Lighvani AA, Frucht DM, Jankovic D, Yamane H, et al. (2001). T-bet is rapidly induced by interferon-gamma in lymphoid and myeloid cells. Proc. Natl. Acad. Sci. U. S. A. 98: 15137-15142.

Lohning M, Richter A and Radbruch A (2002). Cytokine memory of T helper lymphocytes. Adv. Immunol. 80: 115-181.

Lovett-Racke AE, Rocchini AE, Choy J, Northrop SC, et al. (2004). Silencing T-bet defines a critical role in the differentiation of autoreactive T lymphocytes. Immunity 21: 719-731.

Mariani L, Lohning M, Radbruch A and Hofer T (2004). Transcriptional control networks of cell differentiation: insights from helper T lymphocytes. Prog. Biophys. Mol. Biol. 86: 45-76.

Mullen AC, Hutchins AS, High FA, Lee HW, et al. (2002). Hlx is induced by and genetically interacts with T-bet to promote heritable $\mathrm{T}(\mathrm{H}) 1$ gene induction. Nat. Immunol. 3: 652-658.

Murphy KM and Reiner SL (2002). The lineage decisions of helper T cells. Nat. Rev. Immunol. 2: 933-944.

Nakanishi K, Yoshimoto T, Tsutsui H and Okamura H (2001). Interleukin-18 regulates both Th1 and Th2 responses. Annu. Rev. Immunol. 19: 423-474.

O'Garra A (2000). T-cell differentiation: Commitment factors for T helper cells. Curr. Biol. 10: 492-494.

O'Garra A and Arai N (2000). The molecular basis of T helper 1 and T helper 2 cell differentiation. Trends Cell Biol. 10: 542-550.

Ouyang W, Lohning M, Gao Z, Assenmacher M, et al. (2000). Stat6-independent GATA-3 autoactivation directs IL-4independent $\mathrm{Th} 2$ development and commitment. Immunity 12: 27-37.

Ranganath S and Murphy KM (2001). Structure and specificity of GATA proteins in Th2 development. Mol. Cell Biol. 21: $2716-2725$

Robinson DS and Lloyd CM (2002). Asthma: T-bet - a master controller? Curr. Biol. 12: R322-R324.

Romagnani S (1992). Human TH1 and TH2 subsets: regulation of differentiation and role in protection and immunopathology. Int. Arch. Allergy Immunol. 98: 279-285.

Romagnani S (2000). The role of lymphocytes in allergic disease. J. Allergy Clin. Immunol. 105: 399-408.

Smits HH, van Rietschoten JG, Hilkens CM, Sayilir R, et al. (2001). IL-12-induced reversal of human Th2 cells is accompanied by full restoration of IL-12 responsiveness and loss of GATA-3 expression. Eur. J. Immunol. 31: 1055-1065.

Szabo SJ, Kim ST, Costa GL, Zhang X, et al. (2000). A novel transcription factor, T-bet, directs Th1 lineage commitment. Cell 100: 655-669.

Szabo SJ, Sullivan BM, Peng SL and Glimcher LH (2003). Molecular mechanisms regulating Th1 immune responses. 
Annu. Rev. Immunol. 21: 713-758.

Viola JP and Rao A (1999). Molecular regulation of cytokine gene expression during the immune response. J. Clin. Immunol. 19: 98-108.

Wang Q, Liu Y and Mo L (2007a). The Evaluation and Prediction of the Effect of AIDS Therapy. Proceeding of the IEEE/ ICME International Conference on Complex Medical Engineering, Beijing, 1591-1596.

Wang Q, Liu Y and Zhang B (2007b). Economic Strategies in the Issue of Controlling AIDS. Proceeding of the IEEE/ ICME International Conference on Complex Medical Engineering, Beijing, 1601-1608.

Wang Q, Liu Y and Pan X (2008). Atmosphere pollutants and mortality rate of respiratory diseases in Beijing. Sci. Total Environ. 391: 143-148.

Xia L and Zhou C (2007). Phase transition in sequence unique reconstruction. J. Syst. Sci. Complex. 20: 18-29.

Xia LC, Cram JA, Chen T, Fuhrman JA, et al. (2011). Accurate genome relative abundance estimation based on shotgun metagenomic reads. PLoS One 6: e27992.

Yates A, Callard R and Stark J (2004). Combining cytokine signalling with T-bet and GATA-3 regulation in Th1 and Th2 differentiation: a model for cellular decision-making. J. Theor. Biol. 231: 181-196.

Zhou M and Ouyang W (2003). The function role of GATA-3 in Th1 and Th2 differentiation. Immunol. Res. 28: 25-37.

Zhou M, Ouyang W, Gong Q, Katz SG, et al. (2001). Friend of GATA-1 represses GATA-3-dependent activity in CD4+ T cells. J. Exp. Med. 194: 1461-1471.

Zhou W and Nakhleh L (2011). Properties of metabolic graphs: biological organization or representation artifacts? BMC Bioinformatics 12: 132.

Zu H, Wang Q, Dong M, Ma L, et al. (2012). Compressed sensing based fixed-point DCT image encoding. Adv. Comput. Math. Appl. 2: 259-262. 